\title{
OBSERVATION OF GAMMA RAYS FROM THE GALACTIC CENTER WITH THE MAGIC TELESCOPE
}

J. Albert ${ }^{1}$ E. Aliu,${ }^{2}$ H. Anderhub,${ }^{3}$ P. Antoranz, ${ }^{4}$ A. Armada,${ }^{2}$ M. Asensio, ${ }^{4}$ C. Baixeras, ${ }^{5}$ J. A. Barrio, ${ }^{4}$ M. Bartelt,${ }^{6}$ H. Bartko, ${ }^{7}$ D. Bastieri, ${ }^{8}$ R. Bavikadi, ${ }^{9}$ W. Bednarek,,${ }^{10}$ K. Berger, ${ }^{1}$ C. Bigongiari, ${ }^{8}$ A. Biland, ${ }^{3}$ E. Bisesi, ${ }^{9}$

R. K. Bock, ${ }^{7}$ T. Bretz, ${ }^{1}$ I. Britvitch, ${ }^{3}$ M. Camara, ${ }^{4}$ A. Chilingarian, ${ }^{11}$ S. Ciprini, ${ }^{12}$ J. A. Coarasa, ${ }^{7}$ S. Commichau, ${ }^{3}$ J. L. Contreras, ${ }^{4}$ J. Cortina, ${ }^{2}$ V. Curtef,${ }^{6}$ V. Danielyan, ${ }^{11}$ F. Dazzi, ${ }^{8}$ A. De Angelis, ${ }^{9}$ R. De los Reyes, ${ }^{4}$ B. De Lotto, ${ }^{9}$ E. Domingo-Santamaría, ${ }^{2}$ D. Dorner, ${ }^{1}$ M. Doro, ${ }^{8}$ M. Errando, ${ }^{2}$ M. Fagiolini, ${ }^{13}$ D. Ferenc, ${ }^{14}$ E. Fernández, ${ }^{2}$ R. Firpo, ${ }^{2}$ J. Flix, ${ }^{2}$ M. V. Fonseca, ${ }^{4}$ L. Font ${ }^{5}$ N. Galante, ${ }^{13}$ M. GarczarczyK, ${ }^{7}$ M. Gaug, ${ }^{2}$ M. Giller, ${ }^{10}$ F. Goebel, ${ }^{7}$ D. Hakobyan, ${ }^{11}$ M. Hayashida, ${ }^{7}$ T. Hengstebeck, ${ }^{15}$ D. Höhne, ${ }^{1}$ J. Hose, ${ }^{7}$ P. Jacon, ${ }^{10}$ O. Kalekin, ${ }^{15}$ D. Kranich, ${ }^{3,14}$ A. Laille ${ }^{14}$ T. Lenisa, ${ }^{9}$ P. Liebing, ${ }^{7}$ E. Lindfors, ${ }^{12}$ F. Longo, ${ }^{16}$ J. López, ${ }^{2}$ M. López, ${ }^{4}$ E. Lorenz, ${ }^{3,7}$ F. Lucarelli, ${ }^{4}$ P. Majumdar, ${ }^{7}$ G. Maneva, ${ }^{17}$ K. Mannheim, ${ }^{1}$ M. Mariotti,${ }^{8}$ M. Martínez, ${ }^{2}$ K. Mase, ${ }^{7}$ D. Mazin, ${ }^{7}$ M. Merck, ${ }^{1}$

M. Meucci, ${ }^{13}$ M. Meyer, ${ }^{1}$ J. M. Miranda, ${ }^{4}$ R. Mirzoyan, ${ }^{7}$ S. Mizobuchi, ${ }^{7}$ A. Moralejo, ${ }^{7}$ K. Nilsson, ${ }^{12}$ E. Oña-Wilhelmi, ${ }^{2}$ R. Orduña,${ }^{5}$ N. Otte, ${ }^{7}$ I. Oya, ${ }^{4}$ D. Paneque, ${ }^{7}$ R. Paoletti,${ }^{13}$ M. Pasanen, ${ }^{12}$ D. Pascoli,${ }^{8}$ F. Pauss, ${ }^{3}$ N. Pavel ${ }^{15}$ R. Pegna, ${ }^{13}$ L. Peruzzo, ${ }^{8}$ A. Piccioli, ${ }^{13}$ E. Prandini, ${ }^{8}$ J. Rico, ${ }^{2}$ W. Rhode, ${ }^{6}$ B. Riegel, ${ }^{1}$ M. Rissi, ${ }^{3}$ A. Robert ${ }^{5}$ S. Rügamer, ${ }^{1}$ A. Saggion,${ }^{8}$ A. Sánchez,${ }^{5}$ P. Sartori, ${ }^{8}$ V. Scalzotto, ${ }^{8}$ R. Schmitt, ${ }^{1}$ T. Schweizer, ${ }^{15}$ M. Shayduk, ${ }^{15}$ K. Shinozaki, ${ }^{7}$ S. N. Shore, ${ }^{18}$ N. Sidro, ${ }^{2}$ A. Sillanpää, ${ }^{12}$ D. Sobczynska, ${ }^{10}$ A. Stamerra, ${ }^{13}$ A. Stepanian, ${ }^{19}$ L. S. Stark, ${ }^{3}$ L. Takalo, ${ }^{12}$ P. Temnikov, ${ }^{17}$ D. Tescaro, ${ }^{2}$ M. Teshima, ${ }^{7}$ N. Tonello, ${ }^{7}$ A. Torres, ${ }^{5}$ D. F. Torres,${ }^{2,20}$ N. Turini, ${ }^{13}$ H. Vankov, ${ }^{17}$ A. Vardanyan, ${ }^{11}$ V. Vitale, ${ }^{9}$ R. M. Wagner, ${ }^{7}$ T. Wibig, ${ }^{10}$ W. Wittek, ${ }^{7}$ and J. Zapatero ${ }^{5}$ Received 2005 December 14; accepted 2006 January 12; published 2006 January 25

\section{ABSTRACT}

Recently, the Galactic center has been reported to be a source of very high energy (VHE) $\gamma$-rays by the CANGAROO, VERITAS, and HESS experiments. The energy spectra as measured by these experiments show substantial differences. In this Letter we present MAGIC observations of the Galactic center, resulting in the detection of a differential $\gamma$-ray flux consistent with a steady, hard-slope power law, described as $d N_{\gamma} /(d A d t d E)=(2.9 \pm 0.6) \times 10^{-12}(E / \mathrm{TeV})^{-2.2 \pm 0.2} \mathrm{~cm}^{-2} \mathrm{~s}^{-1} \mathrm{TeV}^{-1}$. The $\gamma$-ray source is centered at $(\mathrm{R} . \mathrm{A}$. , decl. $)=\left(17^{\mathrm{h}} 45^{\mathrm{m}} 20^{\mathrm{s}},-29^{\circ} 2^{\prime}\right)$. This result confirms the previous measurements by the HESS experiment and indicates a steady source of $\mathrm{TeV} \gamma$-rays. We briefly describe the observational technique used and the procedure implemented for the data analysis, and we discuss the results in the perspective of different models proposed for the acceleration of the VHE $\gamma$-rays.

Subject headings: acceleration of particles — Galaxy: center — gamma rays: observations

\section{INTRODUCTION}

The Galactic center (GC) region contains many remarkable objects that may be responsible for high-energy processes generating $\gamma$-rays (Aharonian \& Neronov 2005; Atoyan \& Dermer 2004). The GC is rich in massive stellar clusters with up to 100 OB stars (Morris \& Serabyn 1996), immersed in a dense

\footnotetext{
${ }^{1}$ Universität Würzburg, D-97074 Würzburg, Germany.

${ }^{2}$ Institut de Física d'Altes Energies, Edifici Cn., E-08193 Bellaterra (Barcelona), Spain.

${ }^{3}$ ETH Zürich, CH-8093 Hönggerberg, Switzerland.

${ }^{4}$ Universidad Complutense, E-28040 Madrid, Spain.

${ }^{5}$ Universitat Autònoma de Barcelona, E-08193 Bellaterra, Spain.

${ }^{6}$ Universität Dortmund, D-44227 Dortmund, Germany.

${ }^{7}$ Max-Planck-Institut für Physik, D-80805 München, Germany; correspondence should be addressed to H. Bartko, hbartko@mppmu.mpg.de.

${ }^{8}$ Università di Padova and INFN, I-35131 Padua, Italy.

${ }^{9}$ Università di Udine and INFN Trieste, I-33100 Udine, Italy.

${ }^{10}$ University of Łódź, PL-90236 Łódź, Poland.

${ }^{11}$ Yerevan Physics Institute, AM-375036 Yerevan, Armenia.

${ }^{12}$ Tuorla Observatory, FI-21500 Piikkiö, Finland.

${ }^{13}$ Università di Siena and INFN Pisa, I-53100 Siena, Italy.

${ }^{14}$ University of California, Davis, CA 95616-8677.

${ }^{15}$ Humboldt-Universität zu Berlin, D-12489 Berlin, Germany.

${ }^{16}$ Università di Trieste and INFN Trieste, I-34100 Trieste, Italy.

${ }^{17}$ Institute for Nuclear Research and Nuclear Energy, BG-1784 Sofia, Bulgaria.

${ }^{18}$ Università di Pisa and INFN Pisa, I-56126 Pisa, Italy.

${ }^{19}$ Deceased, formerly Crimean Astrophysical Observatory, Ukraine.

${ }^{20}$ Institut de Ciències de 1'Espai, E-08193 Bellaterra (Barcelona), Spain.
}

gas. There are young supernova remnants, e.g., Sgr A East, and nonthermal radio arcs (LaRosa et al. 2000). The dynamical center of the Milky Way is associated with the compact radio source Sgr A*, which is believed to be a massive black hole (Morris \& Serabyn 1996; Schödel et al. 2002).

EGRET has detected a strong source in the direction of the GC, 3EG J1745-2852 (Mayer-Hasselwander et al. 1998; Hartman et al. 1999), which has a broken power-law energy spectrum extending up to at least $10 \mathrm{GeV}$, with a spectral index of 1.3 below the break at a few GeV. Assuming a distance of the GC of $8.5 \mathrm{kpc}$, the $\gamma$-ray luminosity of this source is very large, $2.2 \times 10^{37} \mathrm{ergs} \mathrm{s}^{-1}$, which is equivalent to about 10 times the $\gamma$-ray flux from the Crab Nebula. However, an independent analysis of the EGRET data (Hooper \& Dingus 2002) indicates a point source whose position is different from the GC at a confidence level beyond $99.9 \%$. This was recently sustained by Pohl (2005).

In VHE $\gamma$-rays, the GC has been observed by CANGAROO, VERITAS, and HESS (Tsuchiya et al. 2004; Kosack et al. 2004; Aharonian et al. 2004). The energy spectra as measured by these experiments show substantial differences. This might be due to different sky integration regions of the signal or a source variability at a timescale of about 1 year.

\section{OBSERVATIONS}

MAGIC (Major Atmospheric Gamma Imaging Cerenkov telescope; Baixeras et al. 2004; Cortina et al. 2005) is currently 
TABLE 1

DATA SET

\begin{tabular}{lccccc}
\hline \hline Period & Date & $\begin{array}{c}\text { ZA } \\
(\mathrm{deg})\end{array}$ & $\begin{array}{c}\text { Time } \\
(\mathrm{hr})\end{array}$ & $\begin{array}{c}\text { Events } \\
\left(10^{6}\right)\end{array}$ & Obs. Mode \\
\hline I ........ & 2004 Sep & $62-68$ & 2 & 0.8 & ON \\
II ....... & 2005 May & $58-62$ & 7 & 2.8 & Wobble \\
III ...... & 2005 Jun/Jul & $58-62$ & $17 / 12$ & $6.4 / 5.0$ & ON/OFF \\
\hline
\end{tabular}

Note. - Data set per observation period of the GC. The fourth column states the effective observation time, and the fifth column states the events after image cleaning.

the largest single-dish Imaging Air Cerenkov Telescope (IACT) in operation. Located on the Canary Island La Palma (N28.8, $\mathrm{W} 17.8,2200 \mathrm{~m}$ above sea level), it has a $17 \mathrm{~m}$ diameter tessellated parabolic mirror, supported by a lightweight carbonfiber frame. It is equipped with a high quantum efficiency 576 pixel 3.5 field-of-view photomultiplier camera. The analog signals are transported via optical fibers to the trigger electronics and the 300 MSamples $\mathrm{s}^{-1}$ FADC readout system.

At $\mathrm{La}$ Palma, the GC $\left(17^{\mathrm{h}} 45^{\mathrm{m}} 36^{\mathrm{s}},-28^{\circ} 56^{\prime}\right)$ culminates at about $58^{\circ}$ zenith angle (ZA). The star field around the GC is nonuniform. In the region R.A. $>$ R.A. ${ }_{\mathrm{GC}}+4.7$, the star field is brighter. Within a distance of $1^{\circ}$ from the $\mathrm{GC}$, there are no stars brighter than 8 mag.

The MAGIC observations were carried out in the ON/OFF mode as well as in the false-source tracking (wobble) mode (Fomin et al. 1994). The sky directions (W1, W2) to be tracked in the wobble mode are chosen such that in the camera, the star field relative to the source position (GC) is similar to the star field relative to the mirror source position (antisource position): $\mathrm{W} 1 / \mathrm{W} 2=\left(\mathrm{R} . \mathrm{A} \cdot{ }_{\mathrm{GC}}\right.$, decl. $\left._{\mathrm{GC}} \pm 0.4\right)$. During one wobble mode data taking, $50 \%$ of the data is taken at $\mathrm{W} 1$, and $50 \%$ at W2, switching between the two positions every 20 minutes. Dedicated OFF data have been taken, with a sky field similar to that of the $\mathrm{ON}$ region. The OFF region is centered at the Galactic plane, $\mathrm{GC}_{\mathrm{OFF}}=\left(17^{\mathrm{h}} 51^{\mathrm{m}} 12^{\mathrm{s}},-26^{\circ} 52^{\prime} 00^{\prime \prime}\right)$. In the same night, OFF data were taken directly before and after the $\mathrm{ON}$ observations under the same weather conditions and with the same hardware setup. The observations were scheduled such that the ON and OFF observations have similar ZA range and distribution. After initial observations in 2004 September, the GC was observed for a total of about $24 \mathrm{hr}$ in the period 2005 May-July. Table 1 summarizes the data taken.

\section{DATA ANALYSIS}

The data analysis has been carried out using the standard MAGIC analysis and reconstruction software (Bretz \& Wagner 2003), the first step of which involves the calibration of the raw data (Gaug et al. 2005). After calibration, image cleaning tail cuts of 10 photoelectrons for core pixels and 5 photoelectrons for boundary pixels have been applied (Fegan 1997). These tail cuts are accordingly scaled for the larger size of the outer pixels of the MAGIC camera. The camera images are parameterized by image parameters (Hillas 1985).

In this analysis, a custom implementation (Bock et al. 2004) of the Random Forest (RF) method (Breiman 2001) was applied for the $\gamma$ /hadron separation and the energy estimation (Fegan 1997). In the RF method, to each event several independent decision trees (series of cuts) are applied. By combining the results of the individual decision trees, two parameters are calculated: the estimated energy and the parameter "hadronness," which is a measure of the probability that the event is not $\gamma$ like. The trees of the RF are generated by means of training

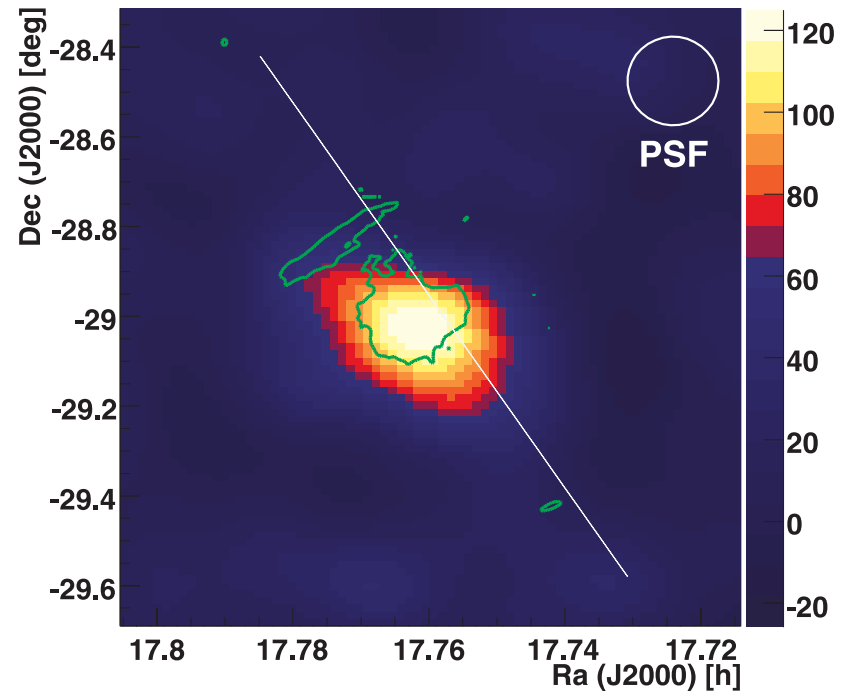

FIG. 1.-Smoothed sky map of $\gamma$-ray candidates (background-subtracted) in the direction of the Galactic center for SIZE $\geq 300$ photoelectrons (corresponding to an energy threshold of about $1 \mathrm{TeV}$ ). Overlaid are green contours (0.3 $\mathrm{Jy} \mathrm{beam}^{-1}$ ) of $90 \mathrm{~cm}$ VLA (BCD configuration) radio data (LaRosa et al. 2000). The white line shows the Galactic plane.

samples for the different classes: A sample of Monte Carlogenerated $\gamma$-ray showers was used together with about $1 \%$ randomly selected events drawn from the measured OFF data. The Monte Carlo (MC) $\gamma$-ray showers were generated between $58^{\circ}$ and $68^{\circ} \mathrm{ZA}$ with energies between $10 \mathrm{GeV}$ and $30 \mathrm{TeV}$. For the analysis of the 2004 September data set, the RF cuts were determined using a subset of Galactic OFF data as background. The source-position-independent image parameters SIZE, WIDTH, LENGTH, CONC (Hillas 1985), and the third moment of the photoelectron distribution along the major image axis, as well as the source-position-dependent parameter DIST (Hillas 1985), were selected to parameterize the shower images. The $\gamma$ ray sample is defined by selecting showers with a hadronness below a specified value. An independent sample of MC $\gamma$-ray showers was used to determine the efficiency of the cuts.

The analysis at high zenith angles was developed and verified using Crab data with a ZA around $60^{\circ}$. The reconstructed Crab energy spectrum was found to be consistent with other existing measurements (see Fig. 3 below, dot-dashed line).

For each event, the arrival direction of the primary in sky coordinates is estimated by using the DISP method (Fomin et al. 1994; Lessard et al. 2001; Domingo-Santamaria et al. 2005): The arrival direction lies on the major axis of the Hillas ellipse that fits the shower image in the camera at a certain distance (DISP) from the image center of gravity. DISP has been parameterized as a function of the ellipticity as in Lessard et al. (2001). One of the two possible arrival directions is selected using the third moment of the photoelectron distribution along the major image axis. For the sky map calculation, only sourceindependent image parameters are used in the RF training. Figure 1 shows the sky map of $\gamma$-ray candidates (backgroundsubtracted; see, e.g., Rowell 2003) from the GC region (observation periods II/III). It is folded with a two-dimensional Gaussian with a standard deviation of $0{ }^{\circ} 1$ (roughly corresponding to the MAGIC point-spread function [PSF]) and a height of 1. A lower SIZE cut of 300 photoelectrons has been applied, corresponding to an energy threshold of about $1 \mathrm{TeV}$. The sky map is overlaid with contours $\left(0.3 \mathrm{Jy}^{\text {beam }^{-1}}\right)$ of $90 \mathrm{~cm} \mathrm{VLA}$ 


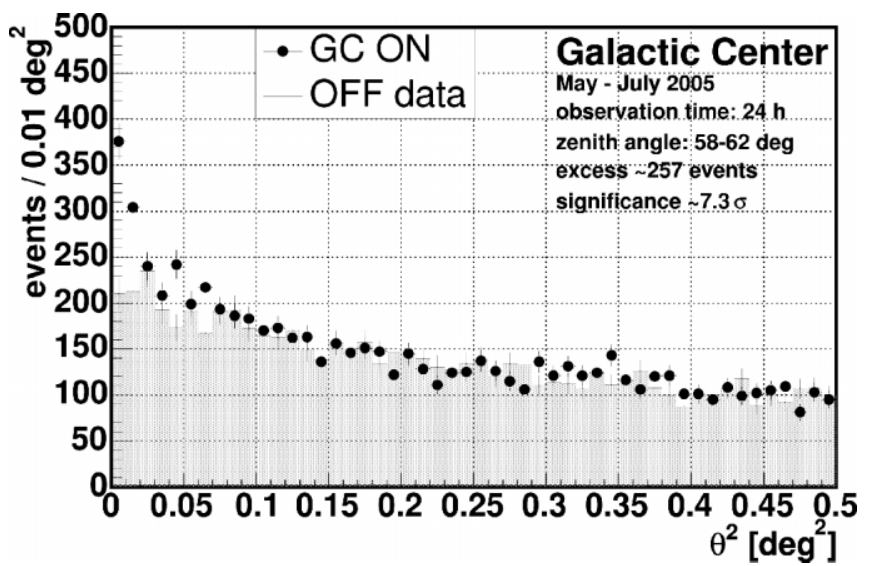

FIG. 2.-Distributions of $\theta^{2}$-values for the source and antisource (see text) for SIZE $\geq 300$ photoelectrons (corresponding to an energy threshold of about $1 \mathrm{TeV})$.

(BCD configuration) radio data from LaRosa et al. (2000). The brightest noncentral source is the Arc. The excess is centered at (R.A., decl.) $=\left(17^{\mathrm{h}} 45^{\mathrm{m}} 20^{\mathrm{s}},-29^{\circ} 2^{\prime}\right)(\mathrm{J} 2000$ coordinates $)$. The present systematic pointing uncertainty is estimated to be $2^{\prime}$ (Bretz et al. 2003). The VHE $\gamma$-ray source G0.9+0.1 (Aharonian et al. 2005) is located inside the MAGIC field of view. It shows a small excess consistent with the low flux reported by Aharonian et al. (2005). The MAGIC excess is not yet statistically significant for the given exposure time.

Figure 2 shows the distribution of the squared angular distance, $\theta^{2}$, between the reconstructed shower direction and the GC excess position (periods II/III). The observed excess in the direction of the GC has a significance of $7.3 \sigma\left(\theta^{2} \leq 0.02\right)$. For large values of $\theta^{2}$, the distributions for ON and OFF data agree well. The source position and the flux level are consistent with the measurement of HESS (Aharonian et al. 2004) within errors.

For the determination of the energy spectrum, the RF was trained, including the source-position-dependent image parameter DIST. For the spectrum determination only, the largest data set (period III) was used. The cut on the hadronness parameter (50\% $\gamma$-efficiency corresponding to an effective area of about $250,000 \mathrm{~m}^{2}$ ) resulted in about 500 excess events with a minimum SIZE of 200 photoelectrons. Figure 3 shows the reconstructed VHE $\gamma$-ray energy spectrum of the GC after the unfolding with the instrumental energy resolution (see Mizobuchi et al. 2005). The differential $\gamma$-ray flux can be well described by a simple power law:

$$
\begin{aligned}
\frac{d N_{\gamma}}{(d A d t d E)}= & (2.9 \pm 0.6) \times 10^{-12} \\
& \times(E / \mathrm{TeV})^{-2.2 \pm 0.2} \mathrm{~cm}^{-2} \mathrm{~s}^{-1} \mathrm{TeV}^{-1}
\end{aligned}
$$

The given errors $(1 \sigma)$ are purely statistical. The systematic error is estimated to be $35 \%$ in the flux level and 0.2 in the spectral index. The dominant systematic error sources (added in quadrature) are the atmospheric model used in the MC simulations (15\%$20 \%$ ) (Bernlöhr 2000), camera (trigger) inefficiency (10\%), uncertainties in light losses in the optical system $(10 \%)$, and the absolute light-to-effective photoelectron conversion (10\%).

Figure 4 shows the reconstructed integral VHE $\gamma$-ray flux above $1 \mathrm{TeV}$ as a function of time. All OFF data are used for each time bin, resulting in some correlation between the time bins. Different observation modi may result in different systematic errors. The flux level is steady within errors in the

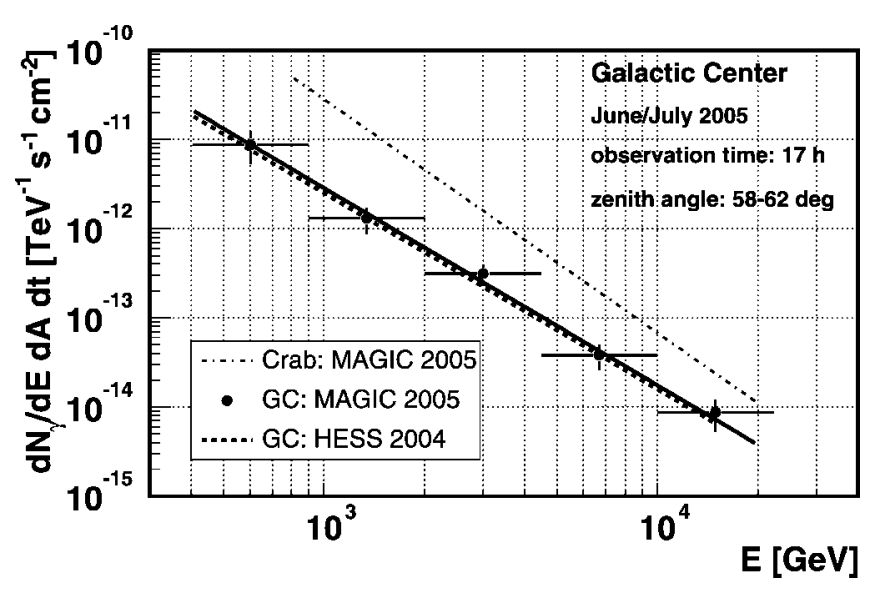

FIG. 3.-Reconstructed VHE $\gamma$-ray energy spectrum of the GC (statistical errors only). The solid line shows the result of a power-law fit to the data points. The dashed line shows the result of the HESS collaboration (Aharonian et al. 2004). The dot-dashed line shows the energy spectrum of the Crab Nebula as measured by MAGIC (Wagner et al. 2005).

timescales explored within these observations, as well as in the 2 year time span between the MAGIC and HESS observations.

\section{DISCUSSION}

Recent observations of $\mathrm{TeV} \gamma$-rays from the GC confirm that this is a very important region for high-energy processes in the Galaxy. Many different objects, able to accelerate particles above $\mathrm{TeV}$ energies, are expected there. The most likely source seems to be the massive black hole identified with Sgr A* due to the directional consistency. A blazar-like relativistic jet originating from the spinning GC black hole might be expected to produce $\mathrm{TeV} \gamma$-rays (Falcke et al. 1993), but flux predictions of this model are on the low side due to an unfavorable orientation of the jet axis. Atoyan \& Dermer (2004) propose that electrons can be accelerated to sufficiently high energies at the termination shock of the subrelativistic wind from the central part of the advection-dominated accretion flow onto the GC black hole, in analogy to the pulsar wind nebulae. The authors suggest that the GeV source observed by EGRET has another origin. This is consistent with the recent determination of the position of the EGRET source 3EG J1746-2851 by Hooper $\&$ Dingus (2002) and Pohl (2005). Other scenarios for the $\gamma$ ray production in the vicinity of Sgr A* have also been found to be consistent with the TeV observations but not with the
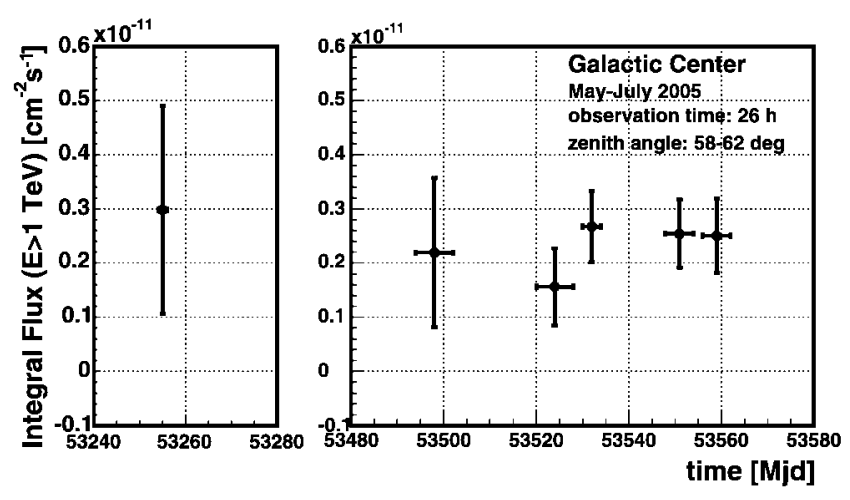

FIG. 4.-Light curve: Reconstructed integral VHE gamma-ray flux above $1 \mathrm{TeV}$ as a function of time. The data are consistent with a steady emission within errors $(1 \sigma)$. 
GeV observations (Aharonian \& Neronov 2005). It is generally expected that $\gamma$-rays produced in such compact source models should show relatively fast variability. Instead, the same level of TeV flux reported by HESS in 2004 and by MAGIC in 2005, and also during their own observation periods extending over a few months, suggests a stable source on a timescale of about 1 year. However, the $\gamma$-ray flux above $2.8 \mathrm{TeV}$ (3.7 $\sigma$ significance) reported by Whipple during the extended period from 1995 through 2003 is a factor 2 larger (Kosack et al. 2004).

The origin of $\gamma$-ray emission in other types of sources is also possible. Pohl (1997) proposed that the GeV emission can be related to the GC radio arc. Crocker et al. (2005; see also Fatuzzo \& Melia 2003) argue for the $\mathrm{GeV}$ and $\mathrm{TeV}$ emission coming from different sites of the shell of the very powerful supernova remnant Sgr A East.

More extended $\gamma$-ray emission might also originate in the interaction of relativistic particles with the soft radiation and matter of the central stellar cluster around the GC. These particles can be accelerated by, e.g., a very energetic pulsar, a $\gamma$-ray burst source, shocks in the winds of the massive stars, or a shell-type supernova remnant (Bednarek 2002; Biermann et al. 2004; Quataert \& Loeb 2005; Crocker et al. 2005; Grasso \& Maccione 2005). If the TeV $\gamma$-rays are produced by leptons scattering off the infrared photons from the dust heated by the UV stellar radiation (Quataert \& Loeb 2005 ), then the $\gamma$-ray power at $\sim 100 \mathrm{GeV}$ should be almost an order of magnitude higher due to scattering of UV radiation. The $\gamma$-ray energy spectrum should steepen between $\sim 0.1$ and $1 \mathrm{TeV}$. Instead, the HESS collaboration reports a simple power-law spectrum between $\sim 0.2$ and $10 \mathrm{TeV}$ (Aharonian et al. 2004). Therefore, hadrons should have energies of about $10^{3} \mathrm{TeV}$. Such hadrons diffuse through the region of the $\mathrm{TeV}$ source $(<7 \mathrm{pc}$; Aharonian et al. 2004) on a timescale of the order of $10^{4} \mathrm{yr}$. Thus, the natural source of relativistic hadrons seems to be the supernova remnant Sgr A East or the energetic pulsar created in the supernova explosion (Crocker et al. 2005; LaRosa et al. 2005; Bednarek 2002). However, this relatively young source of relativistic hadrons cannot be identified with the last $\gamma$-ray burst in the center of our Galaxy if it appeared $\sim 10^{6} \mathrm{yr}$ ago (Biermann et al. 2004).

The GC can also be the brightest source of VHE $\gamma$-rays from particle dark matter annihilation (Prada et al. 2004; Hooper et al. 2004; Flix 2005). Most SUSY dark matter scenarios lead to a cutoff in the $\gamma$-ray energy spectrum below $10 \mathrm{TeV}$. The observed $\gamma$-ray energy spectrum extends up to $20 \mathrm{TeV}$. Thus, most probably the main part of the observed $\gamma$-radiation is not due to dark matter annihilation (Horns 2005). However, an extended $\gamma$-ray source due to dark matter annihilation peaking in the region from $10 \mathrm{GeV}$ to $100 \mathrm{GeV}$ (Elsässer \& Mannheim 2005) cannot be ruled out yet.

\section{CONCLUDING REMARKS}

The MAGIC observations confirm the VHE $\gamma$-ray source at the Galactic center. The measured flux is compatible with the measurement of HESS (Aharonian et al. 2004) within errors. The VHE $\gamma$-ray emission does not show any significant time variability; instead, our measurements affirm a steady emission of $\gamma$-rays from the GC region. The excess is pointlike; its location is spatially consistent with Sgr A* as well as SgrA East.

The nature of the source of the VHE $\gamma$-rays has not yet been identified. Future simultaneous observations with the present Cerenkov telescopes, with the Gamma-Ray Large Area Space Telescope (GLAST), and in the lower energies will provide us with much better information on the source localization and variability of emission. This will shed new light on the nature of the high-energy processes in the GC.

We would like to thank the IAC for the excellent working conditions at the Observatory de los Muchachos in La Palma. This work was supported by the German BMBF and MPG, the Italian INFN, ETH research grant TH-34/04-3, and the Polish MNiI grant 1P03D01028.

\section{REFERENCES}

Aharonian, F., \& Neronov, A. 2005, ApJ, 619, 306

Aharonian, F., et al. 2004, A\&A, 425, L13 2005, A\&A, 432, L25

Atoyan, A., \& Dermer, C. D. 2004, ApJ, 617, L123

Baixeras, C., et al. 2004, Nucl. Instrum. Methods Phys. Res. A, 518, 188

Bednarek, W. 2002, MNRAS, 331, 483

Bernlöhr, K. 2000, Astropart. Phys., 12, 255

Biermann, P. L., Medina Tanco, G., Engel, R., \& Pugliese, G. 2004, ApJ, 604, L29

Bock, R. K., et al. 2004, Nucl. Instrum. Methods Phys. Res. A, 516, 511

Breiman, L. 2001, Machine Learning, 45, 5

Bretz, T., et al. 2003, Proc. 28th Int. Cosmic Ray Conf. (Tsukuba), 2943

Bretz, T., \& Wagner, R. 2003, Proc. 28th Int. Cosmic Ray Conf. (Tsukuba), 2947

Cortina, J., et al. 2005, Proc. 29th Int. Cosmic Ray Conf. (Pune), in press (astro-ph/0508274)

Crocker, R. M., Fatuzzo, M., Jokipii, J. R., Melia, F., \& Volkas, R. R. 2005, ApJ, 622, 892

Domingo-Santamaria, E., et al. 2005, Proc. 29th Int. Cosmic Ray Conf. (Pune), in press (astro-ph/0508274)

Elsässer, D., \& Mannheim, K. 2005, Phys. Rev. Lett., 94, 171302

Falcke, H., Mannheim, K., \& Biermann, P. L. 1993, A\&A, 278, L1

Fatuzzo, M., \& Melia, F. 2003, ApJ, 596, 1035

Fegan, D. J. 1997, J. Phys. G, 23, 1013

Flix, J. 2005, preprint (astro-ph/0505313)

Fomin, V. P., Stepanian, A. A., Lamb, R. C., Lewis, D. A., Punch, M., \& Weekes, T. C. 1994, Astropart. Phys., 2, 137

Gaug, M., et al. 2005, Proc. 29th Int. Cosmic Ray Conf. (Pune), in press (astro$\mathrm{ph} / 0508274)$
Grasso, D., \& Maccione, L. 2005, Astropart. Phys., 24, 273

Hartman, R. C., et al. 1999, ApJS, 123, 79

Hillas, A. M. 1985, Proc. 19th Int. Cosmic Ray Conf. (La Jolla), 3, 445

Hooper, D., de la Calle Perez, I., Silk, J., Ferrer, F., \& Sarkar, S. 2004, J. Cosmol. Astropart. Phys., 9, 2

Hooper, D., \& Dingus, B. 2005, Adv. Space Res., 35, 130

Horns, D. 2005, Phys. Lett. B, 607, 225

Kosack, K., et al. 2004, ApJ, 608, L97

LaRosa, T. N., Brogan, C. L., Shore, S. N., Lazio, T. J., Kassim, N. E., \& Nord, M. E. 2005, ApJ, 626, L23

LaRosa, T. N., Kassim, N. E., Lazio, T. J. W., \& Hyman, S. D. 2000, AJ, 119, 207 (erratum 199, 3145)

Lessard, R. W., Buckley, J. H., Connaughton, V., \& Le Bohec, S. 2001, Astropart. Phys., 15, 1

Mayer-Hasselwander, H. A., et al. 1998, A\&A, 335, 161

Mizobuchi, S., et al. 2005, Proc. 29th Int. Cosmic Ray Conf. (Pune), in press (astro-ph/0508274)

Morris, M., \& Serabyn, E. 1996, ARA\&A, 34, 645

Pohl, M. 1997, A\&A, 317, 441 2005, ApJ, 626, 174

Prada, F., Klypin, A., Flix, J., Martínez, M., \& Simonneau, E. 2004, Phys. Rev. Lett., 93, 241301

Quataert, E., \& Loeb, A. 2005, ApJ, 635, L45

Rowell, G. P. 2003, A\&A, 410, 389

Schödel, R., et al. 2002, Nature, 419, 694

Tsuchiya, K., et al. 2004, ApJ, 606, L115

Wagner, R., et al. 2005, Proc. 29th Int. Cosmic Ray Conf. (Pune), in press (astro-ph/0508244) 